\title{
On the Techniques to Develop Millimetre-Wave Textile Integrated Waveguides Using Rigid Warp Threads
}

\author{
Leticia Alonso-González, Student Member, IEEE, Samuel Ver-Hoeye, Member, IEEE, Carlos Vázquez-Antuña, \\ Miguel Fernández-García and Fernando Las-Heras Andrés, Senior Member, IEEE
}

\begin{abstract}
Two millimetre-wave textile integrated waveguides, which only differ in the employed substrate, have been designed, manufactured and experimentally characterized. Both waveguides have been conceived to be based on the conventional substrate integrated waveguide technology while being fully integrated in textile. The manufactured prototypes have been characterized by using a back to back textile integrated waveguide to rectangular waveguide transition. The theoretically predicted behaviour of the prototypes has been experimentally verified.
\end{abstract}

Index Terms-Textile integrated circuits (TIC), textile integrated waveguide (TIW), smart textile, wearable, millimetrewave.

\section{INTRODUCTION}

D URING the last years, there has been an overwhelming interest in flexible circuits based on textile technology thanks to the possibility of integrating them in special garments or developing comformable structures. In the literature, a variety of solutions to develop Textile Integrated Circuits (TIC) have been proposed, from the embroidery based designs [1-5], to the non-woven based solutions [6-8], including designs based on using several fabrics with different electromagnetic behaviours [9-12], or inkjet printed over textile substrates based prototypes [13-16]. Moreover, recently there has been an increasing interest in the development of TIC based on the Substrate Integrated Waveguide (SIW) technology [17, 18].

Embroidery based prototypes present the advantage of having a reasonable resolution for the details in the design, although this depends on the precision of the automatic embroidery machine. Embroidery based solutions are not conceived for multilayered prototypes, but for groundless planar antennas or inductors, unless this technology is used together with several fabrics with different electromagnetic behaviours.

Non-wovens are fabric-like materials made from different fibers, bonded together by mechanical, chemical, heat or solvent treatment. There are non-wovens with different

This paper is an expanded version from the IEEE MTT-S International Conference on Numerical Electromagnetic and Multiphysics and Optimization for RF, Microwave and Terahertz Applications, Seville, Spain, May 10-12, 2017. This work has been supported by Gobierno de España TEC201572110-EXP, MINECO-17-TEC2016-80815-P and FPU14/00016 grant, and by the Gobierno del Principado de Asturias (PCTI)/FEDER-FSE under projects EQUIP08-06, EQUIP10-31, IDI/2016/000373 and GRUPIN14-114.

The authors are with the Signal Theory and Communications Area, Department of Electrical Engineering, University of Oviedo, Gijón E-33203, Spain (e-mail: alonsoleticia@uniovi.es - lalonso@tsc.uniovi.es). electromagnetic characteristics, for instance, dielectric nonwovens can be used together with conductive inkjet printing technology to add a conductive layer to the circuit. Conductive non-wovens can be used as conductive layouts combined with dielectric stuck on layers to conform multilayered structures.

Designs based on different fabrics have the advantage that they can be multilayered prototypes and each layer can be structured as required, depending on the accuracy of the cutting plotter. The different fabrics are then connected by sewing procedures or dielectric adhesives. The use of adhesives implies not only the risk of the adhesive deterioration due to the wear out, but also, by connecting different fabrics using any method, the thickness of the prototype increases, making it less interesting for certain low-profile wearable applications.

Conductive inkjet printing designs are conceived to be used with dielectric fabrics or non-wovens. This technique presents as much accuracy as the employed mask has to delimit the conductive layout. However, as with the adhesives, the conductive ink can be deteriorated due to the wear out.

Some solutions to develop TIC using SIW technology have been presented in the literature, based on using two conductive fabrics for the top and bottom layers separated by a fabric woven with dielectric material. The conductive vias are performed by using different approaches such as conductive textile seams or brass eyelets.

All the before mentioned techniques to develop TIC are conceived as a post processing of an existing fabric, thus neither they are suitable for being applied during the same process as the fabric is being manufactured, nor the circuit can be fully integrated in the textile. For these reasons, the mentioned solutions for developing TIC are not the best options for large scale production with the already existing industrial textile machinery such as conventional looms with a dobby or jacquard weaving mechanism.

With the aim of developing millimetre-wave SIW fully integrated in textile, a technique based on the weaving technology is proposed in this paper. This technique allows the prototypes to be manufactured in the same process as the fabric is being fabricated, avoiding posterior treatments and making them suitable for large scale production. An initial methodology to design and simulate TIC and, in particular, Textile Integrated Waveguides (TIW), working in a range of frequencies in which the dimensions of the involved threads are comparable to the wavelength, has already been discussed in [19]. Therefore, a parametric three-step based modelling to 
simulate TIW has been presented. The principle of working of this modelling is based on reducing the computational complexity of the initial problem by using electromagnetically equivalent simpler problems.

The work is organize as follows, in Section II, the three-step characterization model used to simplify the simulation of TIC will be introduced. In Section III the employed materials and the proposed design methodology will be described. In Section IV the fabrication process of the manufactured prototypes will be explained, and experimental results will be presented and discussed in Section V.

\section{Characterisation and Simulation of Textile STRUCTURES METHOdOLOGY}

Every woven prototype can be divided into two types of threads, the warp ones and the weft ones, which are perpendicular to each other. The warp direction coincides with the length of the fabric, while the weft direction coincides with the width. Each type of thread can be characterized by its rigidity or composition. The rigidity of the threads depends not only on the material they are composed by, but also on their fabrication process. Therefore, multifilament threads are more flexible, and monofilament threads are more rigid. When, for instance, the warp threads are more rigid than the weft threads, the weft threads tend to conform the woven structure around the warp threads while the warp threads remain straight, or vice versa.

The electromagnetic simulation of multifilament based millimetre-wave TIC suppose a complex computational problem due to the meshing. For this reason, a three-step parametric modelling is proposed and applied to the development of TIW. Figure 1 depicts the three different steps of the modelling and their characteristic relative permittivities.

FILAMENT MODEL
$\varepsilon_{\mathrm{fil}}$$\rightarrow \begin{gathered}\text { MONOF. MODEL } \\ \varepsilon_{\mathrm{mon}}\end{gathered} \rightarrow \begin{gathered}\text { LAYERS MODEL } \\ \varepsilon_{\mathrm{eq}}\end{gathered}$

Fig. 1. Three-step characterization model and characteristic relative permittivities.

\section{A. Filament Model (FM)}

The FM emulates the real composition of a thread, therefore, it is the most computationally complex model. It takes into account the number of filaments which form the thread, the dimensions of each filament and the relative permittivity of the dielectric material from which the filaments have been extruded, denoted by $\varepsilon_{\text {fil }}$.

\section{B. Monofilament Model (MM)}

Due to the air gaps between the filaments which form each thread, an electromagnetically equivalent monofilament thread can be defined whose relative permittivity is reduced and denoted by $\varepsilon_{\text {mon }}$. The $\mathrm{MM}$ is computationally simpler than the FM and easily translatable into a Computer Aided Design (CAD) weaving software for computerized looms.

\section{Layers Model (LM)}

Due to the air gaps between the monofilament threads, an electromagnetically equivalent model can be defined, using an homogenous dielectric substrate whose relative permittivity has been reduced and denoted by $\varepsilon_{\text {eq. }}$. The LM is based on the conventional method to simulate SIW and represents the computationally simplest model.

\section{Millimetre-WAVE TIW Using Rigid WARP THREADS DESIGN}

Two millimetre-wave TIW using rigid warp threads have been designed which only differ in the dielectric substrate. The designs have been denoted by $d s g n_{1}$ and $d s g n_{2}$ respectively. First, the materials have been chosen for their characterization and secondly, the subsequent translation from the FM to the MM and LM is carried out. For the experimental validation a back to back TIW to WR-28 waveguide transition has been designed and manufactured.

\section{A. Characterization of the Materials}

For the design of the textile prototypes, a previous characterization of the chosen materials has been required. For the electrically conductive threads of the prototypes Shieldex yarns have been used, in particular, Shieldex 117f17 1-ply for the warp threads with a linear resistance of $3.9 \Omega / \mathrm{cm}$ and Shieldex $117 f 17$ 2-ply for the weft threads with a linear resistance of 1.3 $\Omega / \mathrm{cm}$. The ply number means the number of initial threads that are twisted around one another to create one single thread. The number before the $f$ symbol means the weight in dtex per ply. The number after the $f$ symbol means the number of filaments per ply. The dtex unit is the most commonly used unit of measure for the linear mass density of fibers and corresponds with the mass in grams per $10 \mathrm{Km}$ of fiber.

For the dielectric parts of the prototypes three different materials have been used. For the dielectric warp threads in both $d s g n_{1}$ and $d s g n_{2}$ polyethylene-terephthalate (commonly known as PET or polyester) 50 dtex with 18 filaments has been used. For the dielectric weft threads in $d s g n_{1}$ high tenacity polyethersulfone (commonly known as PES) 550 dtex with 80 filaments has been used, whereas in $d_{s g n_{2}}$ PET 167 dtex with 36 filaments has been chosen. The three chosen dielectric threads, with their scattered filaments, are depicted in Fig. 2.

During the fabrication process of multifilament based threads, the different filaments tend to be packed taking on a circular-shaped cross section distribution. However, when the threads are in a woven structure, due to the forces working between them, the cross section of the multifilament threads tend to deform. Some approaches to characterize the deformed cross section of these threads have been widely used such as the Pierce's elliptic cross section, the Kemp's racetrack section, the Hearle's section or the Bowshaped geometry [21]. For the conductive and dielectric warp threads, in both designs, the Pierce's elliptic cross section has been used, in which the filaments tend to be packed taking on an elliptic-shaped cross section distribution. For the conductive weft threads Kemp's racetrack section has been found to be the best approximation, which consists of a rectangle enclosed by two semicircular 


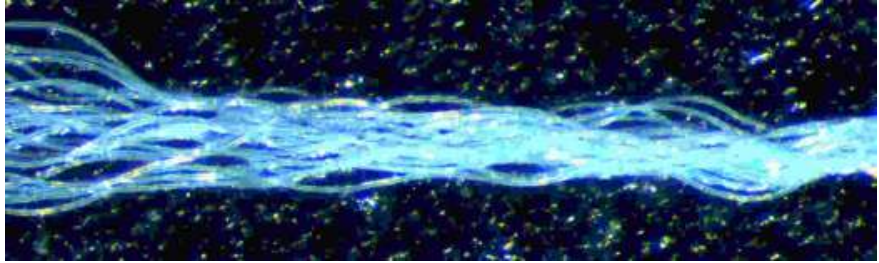

(a)

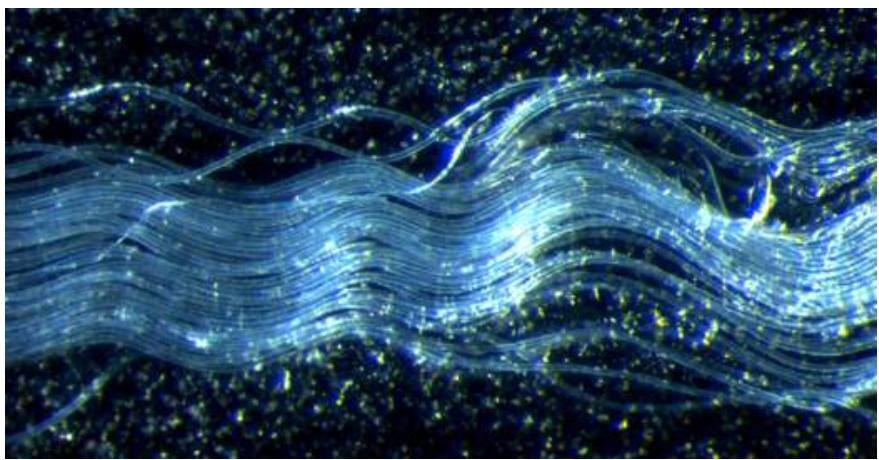

(b)

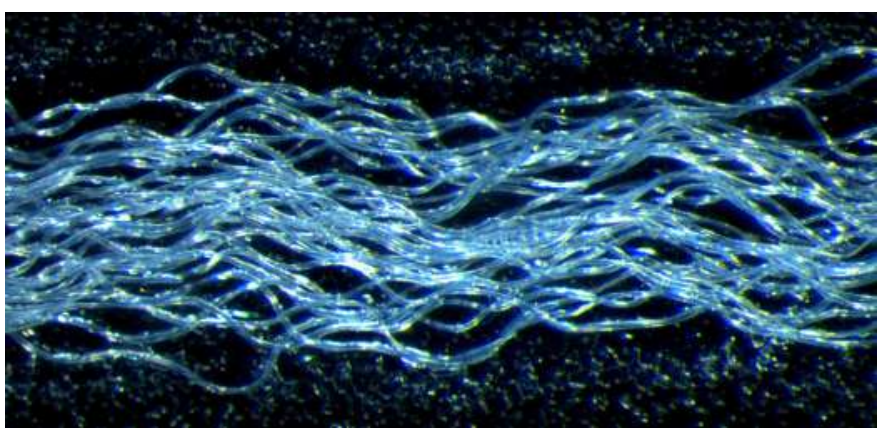

(c)

Fig. 2. Dielectric threads with their scattered filaments. (a) Warp PET thread 50 dtex with 18 filaments (b) Weft high tenacity PES thread 550 dtex with 80 filaments. (c) Weft PET thread 167 dtex with 36 filaments.

ends. However, for the dielectric weft threads in $d s g n_{1}$ (Fig. 2b), Kemp's racetrack section has been used, while for the dielectric weft threads in $d s g n_{2}$ (Fig. 2c) the circular shaped cross section has been kept due to the tenacity of the material. The dimensions of the cross section of each type of thread used in the prototypes are depicted in Table I.

Each filament of PET belonging to the employed dielectric threads has a relative permittivity of $\varepsilon_{\text {fil_PET }}=3.7$ and a density of $\delta_{\text {fil_PET }}=1.5 \mathrm{~g} / \mathrm{cm}^{3}$, whereas each filament of PES has a relative permittivity of $\varepsilon_{\text {fil_PES }}=3.5$ and a density of $\delta_{\text {fil_PES }}=1.5 \mathrm{~g} / \mathrm{cm}^{3}$. With the aim of translating the FM into the MM, finding the three equivalent monofilament threads is required. Taking into account the number of dtex of each thread and the dimensions of the cross section, the proportion of PET, or PES, and air inside the threads can be easily estimated. Therefore, the equivalent relative permittivity of each monofilament thread, $\varepsilon_{\text {mon }}$, can be calculated. For the dielectric warp threads, $25 \%$ of PET has been estimated, whereas for the dielectric weft threads in $d s g n_{1}$ and $d s g n_{2}$, $76 \%$ of PES and $63 \%$ of PET has been estimated, respectively. These porcentages related to the relative permittivities of
TABLE I

DIMENSIONS OF THE CROSS SECTION OF THE MATERIALS USED

\begin{tabular}{|c|c|c|c|c|}
\hline Material & $\begin{array}{c}\text { Thread } \\
\text { direction }\end{array}$ & $\begin{array}{l}\text { Cross } \\
\text { section }\end{array}$ & Dimension & $\begin{array}{l}\text { Value } \\
(\mathrm{mm})\end{array}$ \\
\hline \multirow{4}{*}{ Conductive } & \multirow{2}{*}{ Warp } & \multirow{2}{*}{ Pierce } & Horizontal semi-axis & 0.13 \\
\hline & & & Vertical semi-axis & 0.065 \\
\hline & \multirow{2}{*}{ Weft } & \multirow{2}{*}{ Kemp } & Rectangle length & 0.2 \\
\hline & & & Circumference radio & 0.075 \\
\hline \multirow{4}{*}{$\begin{array}{l}\text { Dielectric } \\
d s g n_{1}\end{array}$} & \multirow{2}{*}{ Warp } & \multirow{2}{*}{ Pierce } & Horizontal semi-axis & 0.075 \\
\hline & & & Vertical semi-axis & 0.055 \\
\hline & \multirow{2}{*}{ Weft } & \multirow{2}{*}{ Kemp } & Rectangle length & 0.2 \\
\hline & & & Circumference radio & 0.075 \\
\hline \multirow{3}{*}{$\begin{array}{l}\text { Dielectric } \\
d s g n_{2}\end{array}$} & \multirow{2}{*}{ Warp } & \multirow{2}{*}{ Pierce } & Horizontal semi-axis & 0.075 \\
\hline & & & Vertical semi-axis & 0.055 \\
\hline & Weft & Circular & Circumference radio & 0.075 \\
\hline
\end{tabular}

eah material, therefore, these relative permittivities have been found to be $\varepsilon_{\text {mon_warp }}=1.72, \varepsilon_{\text {mon_weft_PES }}=2.83$ and $\varepsilon_{\text {mon_weft_PET }}=2.1 \overline{1}$, respectively.

\section{B. Electromagnetic Simulation of the Proposed Modelling}

Once the threads have been characterized, depending on the density of the woven structure, a different $\varepsilon_{\mathrm{eq}}$ is obtained for the LM. The density of the woven prototype is defined by the number of warp threads (commonly known as warp ends) per centimetre. The proposed designs have been simulated using 42 ends per centimetre.

Once the materials have been characterized and the density of the woven structure has been defined, a woven design which emulates the structure of a SIW is required. For the conductive plates of the SIW, two layers composed by the warp and weft conductive threads have been used. The substrate has been emulated using a single layer of dielectric material formed by warp and weft threads. The conductive vias have been emulated using a part of the conductive weft threads crossing the prototype from the top layer to the bottom layer. For this reason, different weft patterns have been required for not only give the prototype a SIW structure, but also to make sure that all the different layers are connected.

Due to the fact that all the warp threads in the designs have a Pierce's cross section, these sections have been modeled as ellipses. For this reason, the paths of the weft threads have been found to be mathematically modeled as ellipse arcs and tangent lines to ellipses. As the model is fully parameterized, finding the tangent points given a pair of ellipses has been required and, therefore, the procedure is explained in detail. Given two generic ellipses, $E_{0}$ and $E_{1}$ respectively, defined by the coordinates of their centres, $E_{C 0}=\left(x_{0}, y_{0}\right)$ and $E_{C 1}=$ $\left(x_{1}, y_{1}\right)$ respectively, and the dimensions of their semiaxes, $\left\{a_{0}, b_{0}\right\}$ and $\left\{a_{1}, b_{1}\right\}$ respectively, the parametric equations to define each ellipse, with $\theta \in[0,2 \pi)$, are given by (1) and (2).

$$
\begin{aligned}
& E_{0}(x)=a_{0} \cos (\theta)+x_{0} \\
& E_{0}(y)=b_{0} \sin (\theta)+y_{0}
\end{aligned}
$$




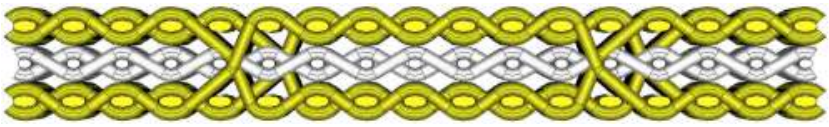

(a)

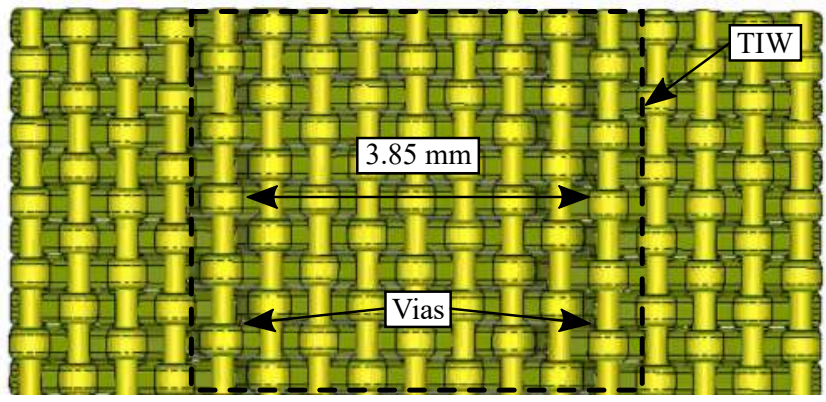

(b)

Fig. 3. Simulated monofilament model of the TIW (conductive material is yellow colored and dielectric materials are white colored). (a) Front view. (b) Top view.

$$
\begin{aligned}
& E_{1}(x)=a_{1} \cos (\theta)+x_{1} \\
& E_{1}(y)=b_{1} \sin (\theta)+y_{1}
\end{aligned}
$$

If a solution exists, then, four different lines are tangent to both ellipses. A line can be defined by two parameters, its slope, $m$, and its intercept, $c$. Thus, with the aim of finding the tangent points between the ellipses and the lines, the equations of the lines are required. By imposing on the line the condition of being tangent to both ellipses, a second order system of equations is generated as depicted in (3).

$$
\left\{\begin{array}{r}
\left.-2 b_{0}^{2} x_{0}-2 m a_{0}^{2}\left(y_{0}-c\right)\right)^{2}+\left(-4\left(b_{0}^{2}+a_{0}^{2} m^{2}\right)\right. \\
\left.\left(x_{0}^{2} b_{0}^{2}+a_{0}^{2}\left(y_{0}-c\right)^{2}-a_{0}^{2} b_{0}^{2}\right)\right)=0 \\
\left.-2 b_{1}^{2} x_{1}-2 m a_{1}^{2}\left(y_{1}-c\right)\right)^{2}+\left(-4\left(b_{1}^{2}+a_{1}^{2} m^{2}\right)\right. \\
\left.\left(x_{1}^{2} b_{1}^{2}+a_{1}^{2}\left(y_{1}-c\right)^{2}-a_{1}^{2} b_{1}^{2}\right)\right)=0
\end{array}\right.
$$

The solution of the mentioned system returns the slopes and the intercepts of the four different lines which are tangent to the ellipses $E_{0}$ and $E_{1}$. From the slopes and the intercepts, the tangent points can be easily calculated. Then, the parametric proposed structure for the MM is depicted in Fig. 3. The dielectric lossy materials have been modeled using as loss tangents $\operatorname{tg}(\delta)_{P E S}=0.001$ and $\operatorname{tg}(\delta)_{P E T}=0.001$ at the working frequency respectively, and the conductive materials have been modeled by an average electrical conductivity of $\sigma_{\text {Shieldex }}=5 \times 10^{4} \mathrm{~S} / \mathrm{m}$.

The proposed structures, for $d s g n_{1}$ and $d s g n_{2}$, have been simulated using a 3D high frequency electromagnetic simulator to estimate the behaviour of the MM and, therefore, predict the values of $\varepsilon_{\mathrm{eq}}$ in the LM for both designs. The equivalent relative permittivities $\varepsilon_{\text {eq_dsgn1 } 1}$ and $\varepsilon_{\text {eq_dsgn2 } 2}$ have been found to be 1.55 in both cases. Even though the composition of the weft threads in both designs is different, a tradeoff exists

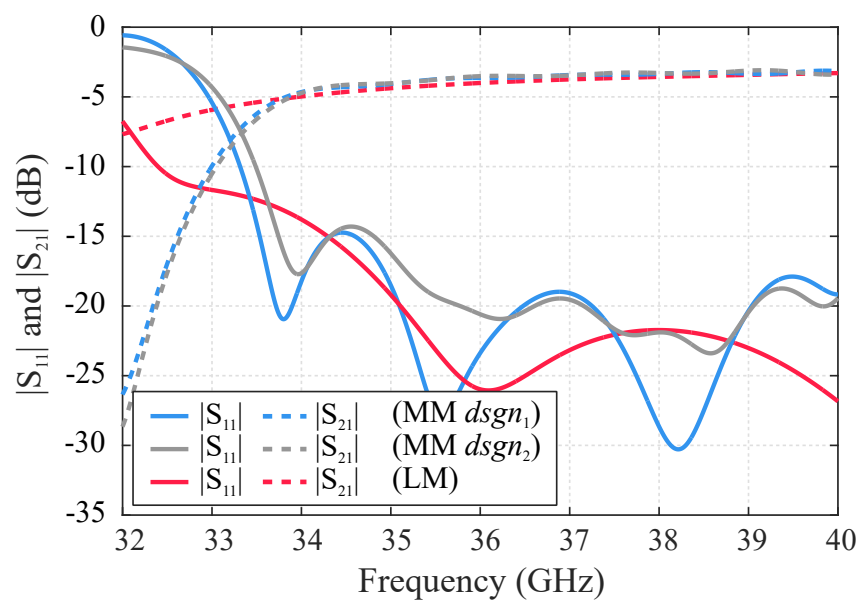

Fig. 4. Comparison between the simulated scattering parameters of the LM and the MM for both designs using a $10 \mathrm{~mm}$ length TIW.

between the difference of the $\varepsilon_{\text {mon_weft }}$ and the dimension of their cross sections. In $d s g n_{1}$, the amount of dielectric material different from air in the substrate is greater than in $d s g n_{2}$, whereas $\varepsilon_{\text {mon_weft_PES }}$ in $d s g n_{1}$ is lower than $\varepsilon_{\text {mon_weft_PET }}$ in $d s g n_{2}$, and vice versa. The electromagnetic simulations of the MM and LM for both designs using a $10 \mathrm{~mm}$ length TIW are depicted in Fig. 4. The different behaviour of the scattering parameters near the cutoff frequency of the TIW is due to the different solvers employed in the simulations. While the LM has been simulated in the frequency domain using a tetrahedral meshing, MM has to be simulated using a time domain technique due to the complexity of the meshing.

\section{Back to Back TIW to WR-28 Waveguide Transition Design}

For the experimental validation of the prototypes, a back to back TIW to WR-28 waveguide has been designed. The transition is based on an air-filled stepped rectangular waveguide with six sections, which have been optimized for maximum coupling between the WR- 28 waveguide and the textile prototypes [20]. The transition has not been symmetrically designed in the $H$-plane, thus it is composed by two parts, a stepped one and a non-stepped one. The port of the transition which is connected to the TIW has been conceived to compress the textile structure using the two mentioned parts for optimum performance. The sketch of the stepped part of the transition is shown in Fig. 5, whereas the dimensions of the steps are depicted in Table II.

The transition has been designed under the assumption of $\varepsilon_{\mathrm{eq}}$ being iqual to 1.55 . However, its behaviour has also been simulated for different equivalent relative permittivities to validate its tolerance to this parameter. The scattering parameters of a $10 \mathrm{~mm}$ length TIW connected to a TIW to WR-28 waveguide transition in each of its ports are presented in Fig. 6.

\section{EXPERIMENTAL RESULTS}

\section{A. Textile Prototype Fabrication}

Two millimetre-wave TIW have been manufactured using an industrial MüGrip loom with a density of 42 ends per 


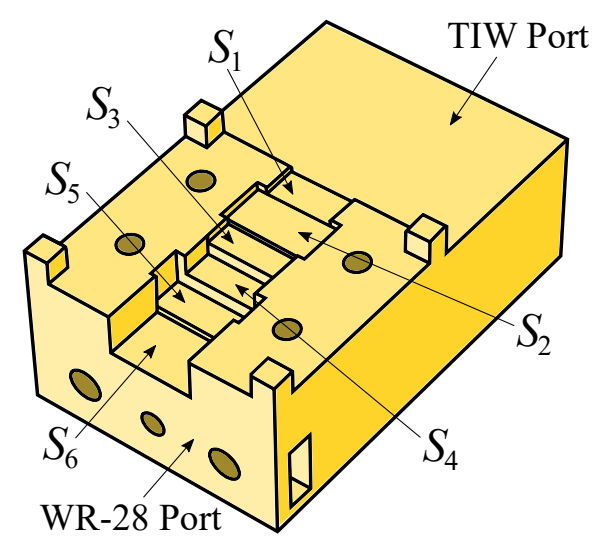

Fig. 5. Inside view of the stepped part of the TIW to WR-28 waveguide transition design.

TABLE II

DIMENSIONS OF THE TIW TO WR-28 WAVEGUIDE TRANSITION

\begin{tabular}{ccccccc}
\hline Step & 1 & 2 & 3 & 4 & 5 & 6 \\
\hline \hline Width $(\mathrm{mm})$ & 5.76 & 7.48 & 6.39 & 6.31 & 7.88 & 7.11 \\
Length $(\mathrm{mm})$ & 2.72 & 4.26 & 2.24 & 2.49 & 2.89 & 3 \\
Height $(\mathrm{mm})$ & 0.36 & 0.26 & 0.69 & 1.78 & 2.97 & 3.56 \\
\hline
\end{tabular}

centimetre, to validate the proposed modelling. Figure 7 depicts the top view of the TIW prototype $\left(d s g n_{1}\right.$ or $d s g n_{2}$ indistinctly) and detail magnifications. The distance between the conductive vias has been found to be $3.85 \mathrm{~mm}$ as predicted. The side views of the manufactured prototypes and corresponding magnifications are depicted in Fig. 8 and Fig. 9 respectively. The mentioned magnifications allows one to perceive the elliptic shaped cross section of the warp threads, especially, in the conductive materials. Moreover, the paths of the weft threads can be easily identified as ellipses arcs and tangent lines to ellipses as predicted, especially in $d s g n_{1}$ due to their high tenacity.

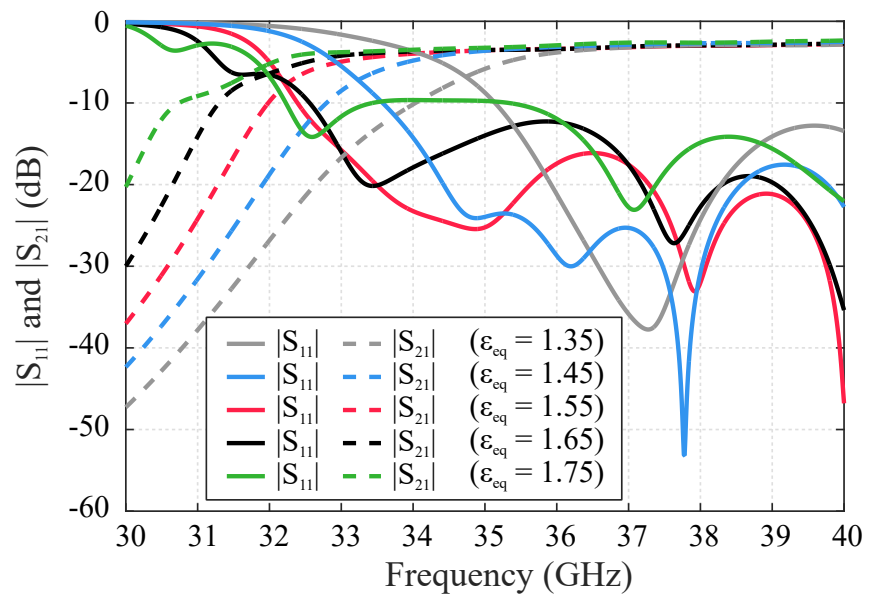

Fig. 6. Simulated scattering parameters of back to back WR-28 waveguide to TIW transition connected to a $10 \mathrm{~mm}$ length TIW using the LM.

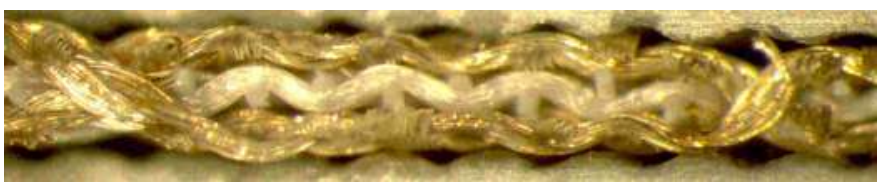

(a)

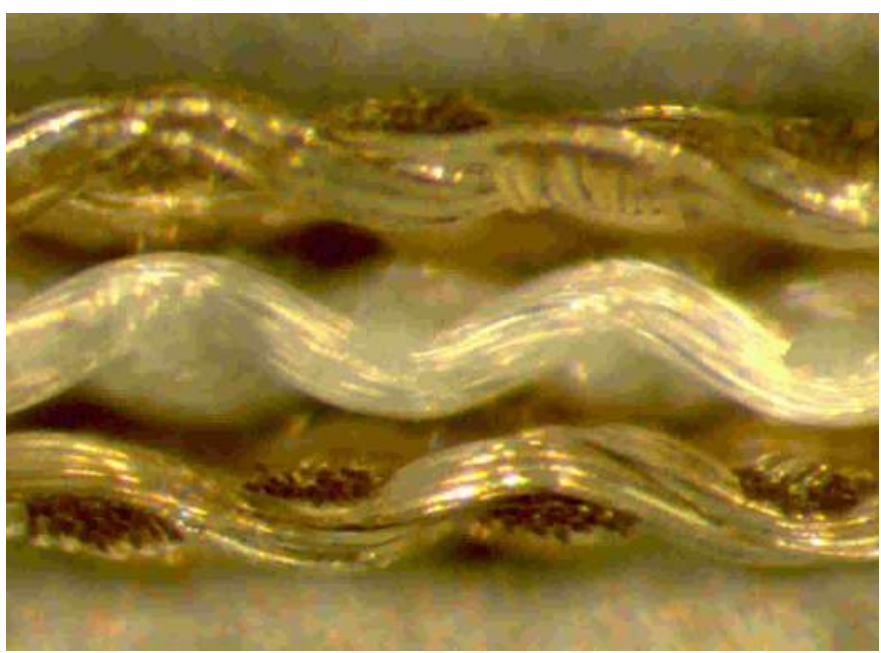

(b)

Fig. 8. TIW prototype using rigid warp threads $\left(d s g n_{1}\right)$ (a) General side view. (b) Magnification of the side view.

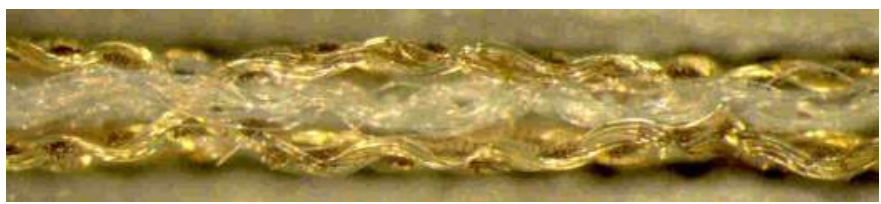

(a)

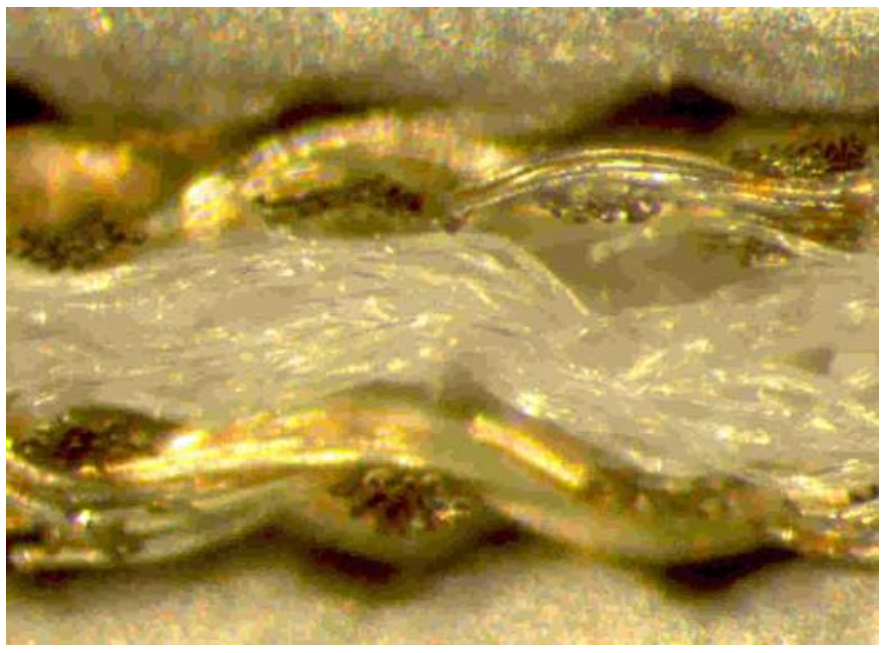

(b)

Fig. 9. TIW prototype using rigid warp threads $\left(d s g n_{2}\right)$ (a) General side view. (b) Magnification of the side view. 


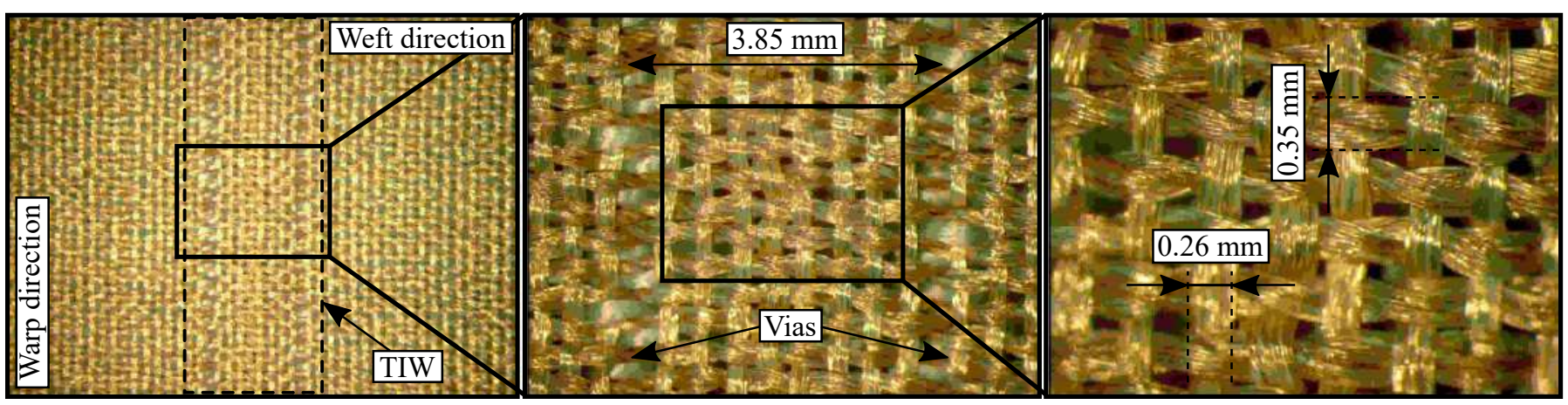

Fig. 7. Top view of the TIW prototype using rigid warp threads ( $d s g n_{1}$ or $d s g n_{2}$ indistinctly) and detailed magnifications.

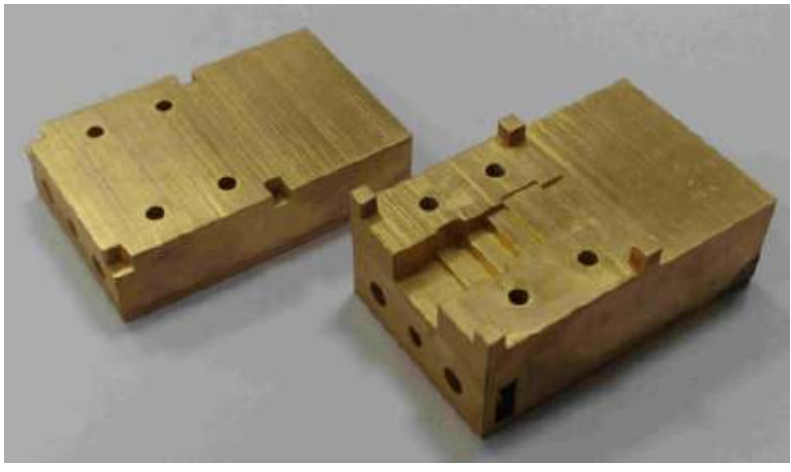

Fig. 10. WR-28 waveguide to TIW manufactured transition.

\section{B. TIW to WR-28 Waveguide Transition Fabrication}

The transition has been manufactured in two different pieces, resulting from dividing it into a stepped part and a nonstepped part. Both parts have been manufactured using a high precision stereolithography process in which a photopolymer resin is progressively cured, layer by layer, with UV radiation exposure, as the solidified part is lifted out of the resin tank.

The inner parts of the transition, where the stepped waveguide is placed, are provided with the required electrical conductivity through the deposition of a thin gold layer in a sputtering process. The manufactured transition is depicted in Fig. 10.

\section{EXPERIMENTAL VALIDATION}

The prototypes have been characterized using the experimental setup presented in Fig. 11. The two ports of the TIW are connected to the TIW to WR-28 waveguide transitions. A pair of R281B waveguide coaxial adapter from WR-28 waveguide to $2.4 \mathrm{~mm}$ from Agilent are required to connect the prototypes to an Agilent N5247A PNA-X vector network analyzer.

Figure 12 represents a comparison between the simulated and measured insertion losses of the TIW $\left(d s g n_{1}\right)$ for three different lengths, $40 \mathrm{~mm}, 50 \mathrm{~mm}$ and $60 \mathrm{~mm}$ respectively. The R281B waveguide coaxial adapters and the TIW to WR-28 waveguide transitions have been included during the calibration, therefore they have not been considered in the measurements. For the simulations, the data from MM model

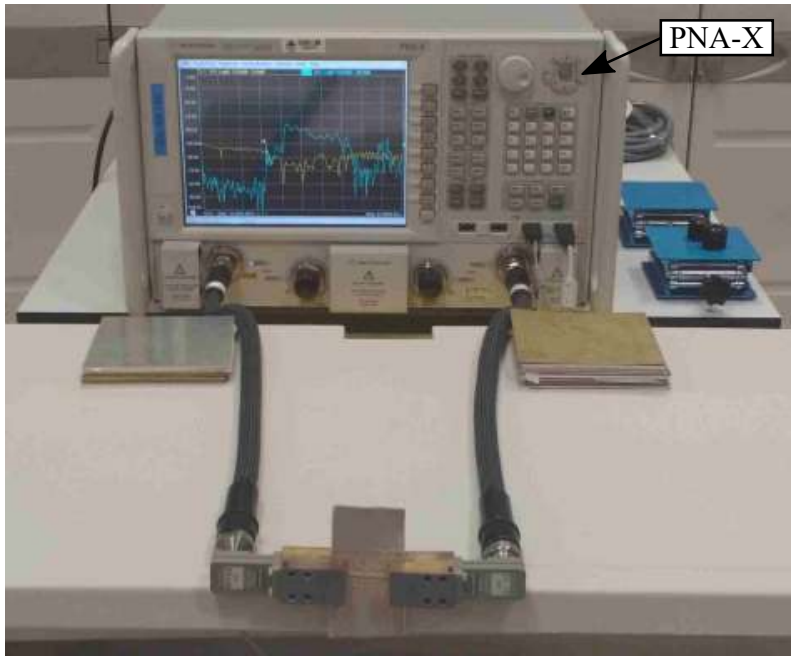

(a)

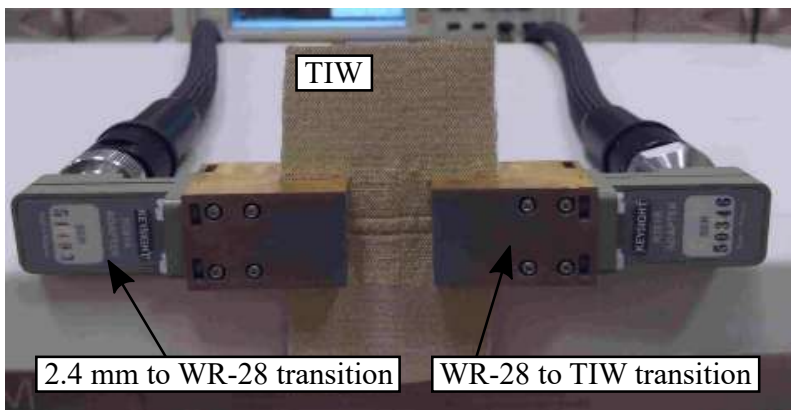

(b)

Fig. 11. Setup for the experimental validation of the TIW prototype using rigid warp threads. (a) General overview. (b) Magnification of the TIW prototype and the transitions.

has been used. A schematic block has been generated with the data of the $10 \mathrm{~mm}$ TIW and it has been replicated for the required lengths using the Advanced Design System software from Keysight Technologies. Figure 13 depicts a comparison between the simulated and measured return losses of the TIW $\left(d s g n_{1}\right)$ for the same three different lengths. Figure 14 and Fig. 15 represent the same comparisons but for $d s g n_{2}$. As predicted in the simulations, both prototypes of TIW present an insertion loss of $3 \mathrm{~dB} / \mathrm{cm}$, especially, due to the ohmmic losses of the conductive materials. 


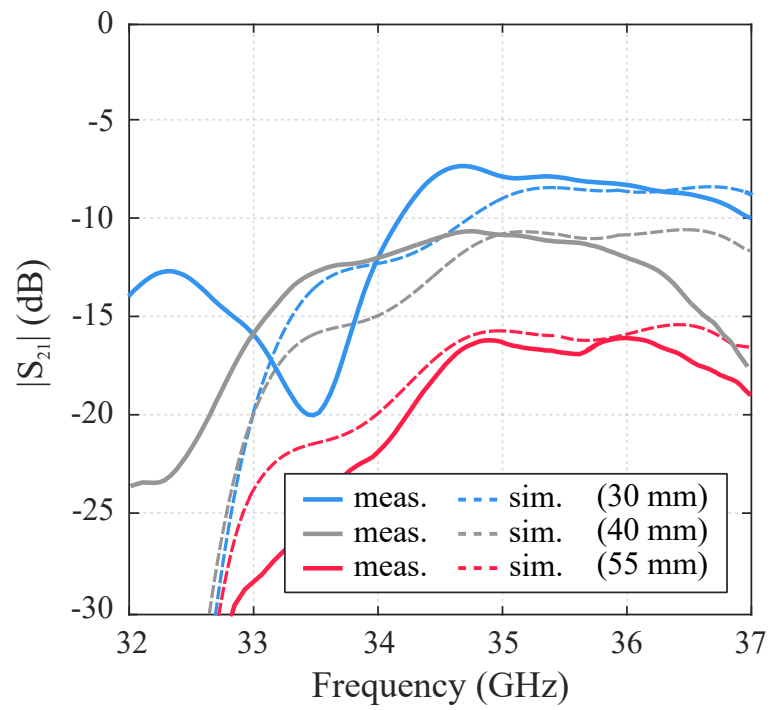

Fig. 12. Simulated and measured values for the insertion losses of the TIW $\left(d s g n_{1}\right)$ for three different lengths.

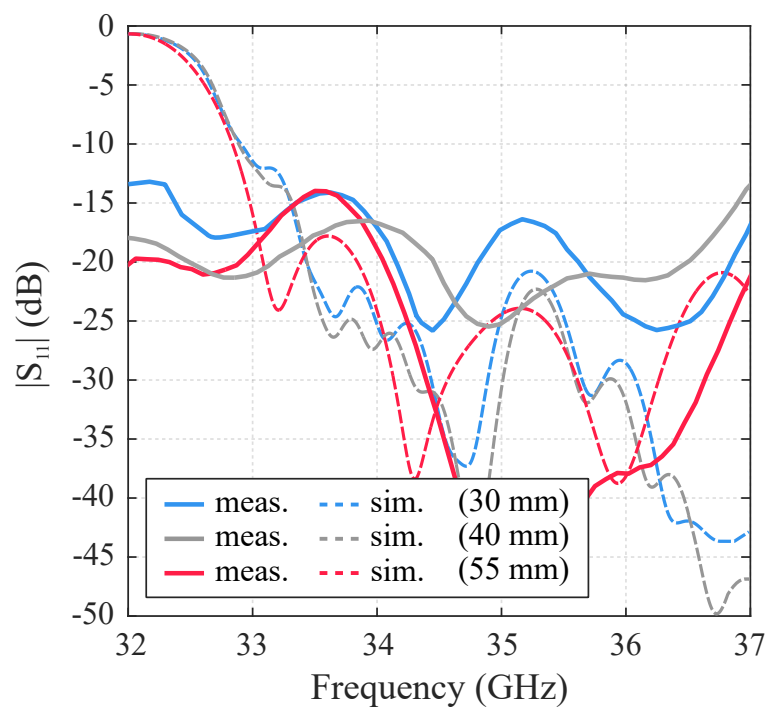

Fig. 13. Simulated and measured values for the return losses of the TIW $\left(d s g n_{1}\right)$ for three different lengths.

\section{CONCLUSION}

A novel technique to develop millimetre-wave TIW using rigid warp threads has been presented. First, a three-step parametric modelling to simulate textile structures based on the reduction of the computational complexity of the problem has been discussed.

To validate the modelling, two TIW, which use different substrates, have been manufactured and experimentally characterized. The theoretically predicted behaviour of the textile structures has been experimentally verified.

\section{ACKNOWLEDGMENT}

The authors would like to thank María García Fernández for her helpful comments with the parametrization.

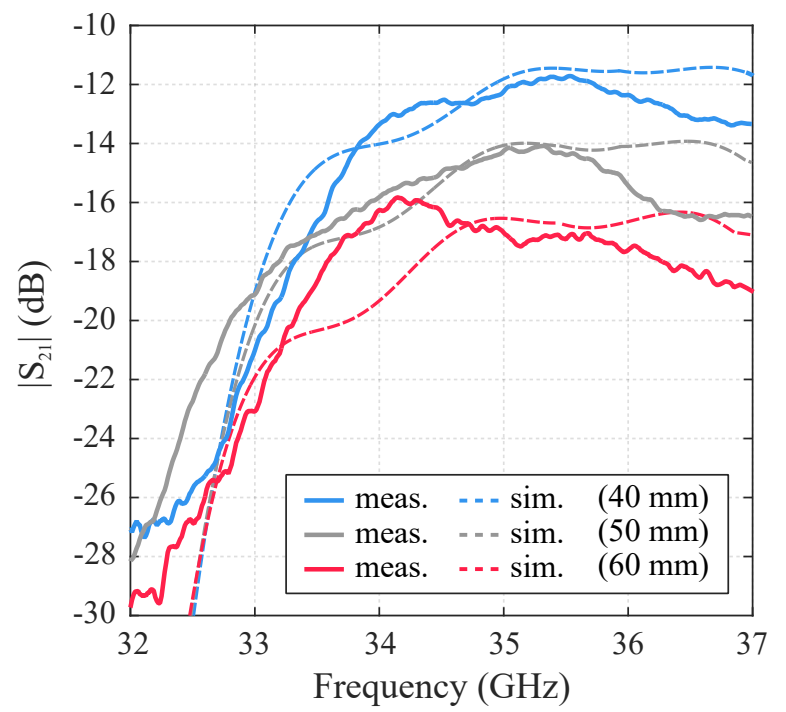

Fig. 14. Simulated and measured values for the insertion losses of the TIW $\left(d s g n_{2}\right)$ for three different lengths.

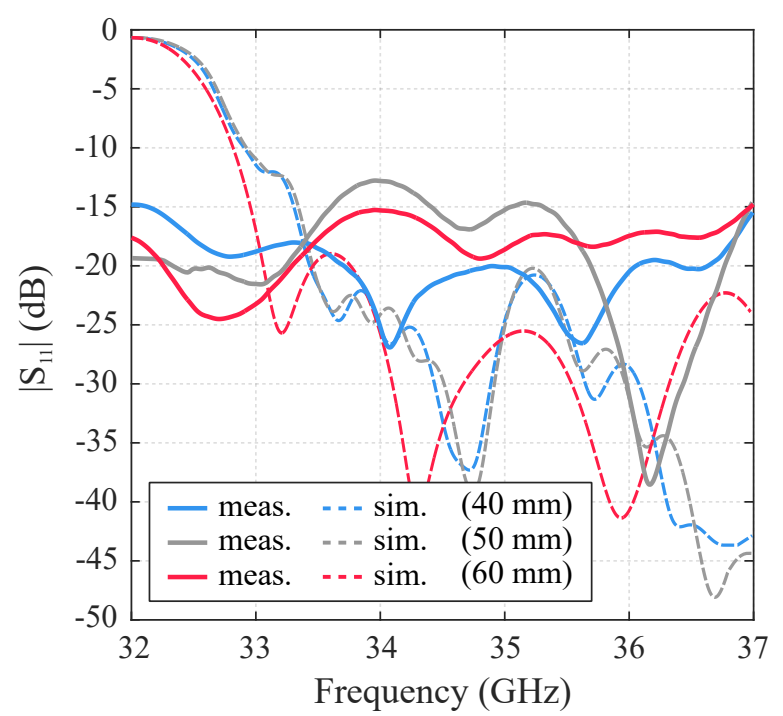

Fig. 15. Simulated and measured values for the return losses of the TIW $\left(d s g n_{2}\right)$ for three different lengths.

\section{REFERENCES}

[1] G. Ginestet et al., Embroidered Antenna-Microchip Interconnections and Contour Antennas in Passive UHF RFID Textile Tags, in IEEE Antennas and Wireless Propagation Letters, vol. 16, no. , pp. 1205-1208, 2017. doi: 10.1109/LAWP.2016.2628086

[2] A. Paraskevopoulos, D. d. S. Fonseca, R. D. Seager, W. G. Whittow, J. C. Vardaxoglou and A. A. Alexandridis, Higher-mode textile patch antenna with embroidered vias for on-body communication, in IET Microwaves, Antennas and Propagation, vol. 10, no. 7, pp. 802-807, 5152016. doi: 10.1049/iet-map.2015.0650

[3] A. Kiourti, C. Lee and J. L. Volakis, Fabrication of Textile Antennas and Circuits With $0.1 \mathrm{~mm}$ Precision, in IEEE Antennas and Wireless Propagation Letters, vol. 15, no., 
pp. 151-153, 2016. doi: 10.1109/LAWP.2015.2435257

[4] Z. Wang, L. Zhang, Y. Bayram and J. L. Volakis, Embroidered Conductive Fibers on Polymer Composite for Conformal Antennas, in IEEE Transactions on Antennas and Propagation, vol. 60, no. 9, pp. 4141-4147, Sept. 2012. doi: 10.1109/TAP.2012.2207055

[5] T. Acti et al., Embroidered Wire Dipole Antennas Using Novel Copper Yarns, in IEEE Antennas and Wireless Propagation Letters, vol. 14, no., pp. 638-641, 2015. doi: 10.1109/LAWP.2014.2371338

[6] Y. Senbokuya and H. Tsunoda, A study on the circular patch antennas using conductive non-woven fiber fabrics, in IEEE Antennas and Propagation Society International Symposium (IEEE Cat. No.02CH37313), 2002, pp. 782784 vol.1. doi: 10.1109/APS.2002.1016459

[7] G. Monti, L. Corchia, E. De Benedetto and L. Tarricone, Wearable logo-antenna for GPSGSM-based tracking systems, in IET Microwaves, Antennas and Propagation, vol. 10, no. 12 , pp. 1332-1338, 917 2016. doi: 10.1049/ietmap.2015.0774

[8] R. K. Shawl, B. R. Longj, D. H. Werner and A. Gavrin, The Characterization of Conductive Textile Materials Intended for Radio Frequency Applications, in IEEE Antennas and Propagation Magazine, vol. 49, no. 3, pp. 28-40, June 2007. doi: 10.1109/MAP.2007.4293934

[9] Xiaoyou Lin, B. C. Seet and F. Joseph, Fabric antenna with body temperature sensing for BAN applications over $5 G$ wireless systems, 2015 9th International Conference on Sensing Technology (ICST), Auckland, 2015, pp. 591595. doi: 10.1109/ICSensT.2015.7438467

[10] S. Kang and C. W. Jung, Wearable fabric antenna on upper arm for MedRadio band applications with reconfigurable beam capability, in Electronics Letters, vol. 51, no. 17, pp. 1314-1316, 820 2015. doi: 10.1049/el.2015.2105

[11] R. Yahya, M. R. Kamarudin, N. Seman and H. U. Iddi, Eye shaped fabric antenna for UWB application, 2013 IEEE Antennas and Propagation Society International Symposium (APSURSI), Orlando, FL, 2013, pp. 228-229. doi: 10.1109/APS.2013.6710775

[12] H. A. Elmobarak Elobaid, S. K. Abdul Rahim, M. Himdi, X. Castel and M. Abedian Kasgari, A Transparent and Flexible Polymer-Fabric Tissue UWB Antenna for Future Wireless Networks, in IEEE Antennas and Wireless Propagation Letters, vol. 16, no. , pp. 1333-1336, 2017. doi: 10.1109/LAWP.2016.2633790

[13] W. G. Whittow et al., Inkjet-Printed Microstrip Patch Antennas Realized on Textile for Wearable Applications, in IEEE Antennas and Wireless Propagation Letters, vol. 13, no. , pp. 71-74, 2014. doi: 10.1109/LAWP.2013.2295942

[14] A. Chauraya et al., Inkjet printed dipole antennas on textiles for wearable communications, in IET Microwaves, Antennas and Propagation, vol. 7, no. 9, pp. 760-767, June 18 2013. doi: 10.1049/iet-map.2013.0076

[15] M. L. Scarpello, I. Kazani, C. Hertleer, H. Rogier and D. Vande Ginste, Stability and Efficiency of Screen-Printed Wearable and Washable Antennas, in IEEE Antennas and Wireless Propagation Letters, vol. 11, no. , pp. 838-841, 2012. doi: 10.1109/LAWP.2012.2207941
[16] M. Akbari, L. Sydänheimo, Y. Rahmat-Sami, J. Virkki and L. Ukkonen, Implementation and performance evaluation of graphene-based passive UHF RFID textile tags, 2016 URSI International Symposium on Electromagnetic Theory (EMTS), Espoo, 2016, pp. 447-449. doi: 10.1109/URSI-EMTS.2016.7571422

[17] R. Moro, S. Agneessens, H. Rogier, A. Dierck and M. Bozzi, Textile Microwave Components in Substrate Integrated Waveguide Technology, in IEEE Transactions on Microwave Theory and Techniques, vol. 63, no. 2, pp. 422-432, Feb. 2015. doi: 10.1109/TMTT.2014.2387272

[18] F. X. Liu, Z. Xu, D. C. Ranasinghe and C. Fumeaux, Textile Folded Half-Mode Substrate-Integrated Cavity Antenna, in IEEE Antennas and Wireless Propagation Letters, vol. 15, no. , pp. 1693-1697, 2016. doi: 10.1109/LAWP.2016.2524458

[19] L. Alonso-González, S. Ver Hoeye, C. Vázquez, M. Fernández, A. Hadarig and F. Las-Heras, Novel parametric electromagnetic modelling to simulate Textile Integrated Circuits, 2017 IEEE MTT-S International Conference on Numerical Electromagnetic and Multiphysics Modeling and Optimization for RF, Microwave, and Terahertz Applications (NEMO), Seville, Spain, 2017, pp. 6769. doi: 10.1109/NEMO.2017.7964189

[20] J. L. Cano, A. Mediavilla and A. R. Perez, Full-Band Air-Filled Waveguide-to-Substrate Integrated Waveguide (SIW) Direct Transition, in IEEE Microwave and Wireless Components Letters, vol. 25, no. 2, pp. 79-81, Feb. 2015. doi: 10.1109/LMWC.2014.2372480

[21] H. U. Jinlian, Structure and mechnics of woven fabrics, Woodhead Publishing in Textiles, The Textile Institute, 2004, pp. 63-66.

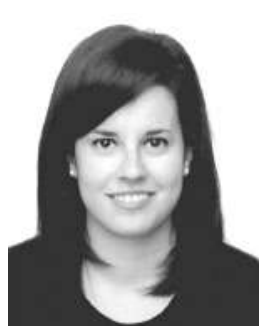

Leticia Alonso-González (S'14) received the M.Sc. degree in telecommunications engineering from the University of Oviedo, Gijón, Spain, in 2014 and she is currently working toward the Ph.D. degree at the University of Oviedo.

Since 2014, she has been working as a researcher in the Signal Theory and Communications Group, University of Oviedo. Her main research effort is focused on the design, simulation and manufacturing techniques to develop microwave and millimetrewave passive circuits and antennas fully integrated

in textile technology.

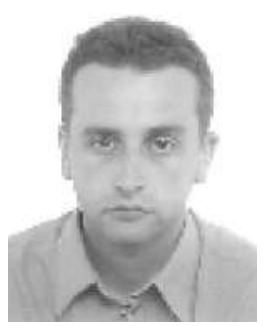

Samuel Ver-Hoeye (M'05) received the M.Sc. degree in electronic engineering from the University of Gent, Gent, Belgium, in 1999, and the Ph.D. degree from the University of Cantabria, Santander, Spain, in 2002 .

He is currently an Associate Professor with the Department of Electrical and Electronic Engineering, University of Oviedo, Gijón, Spain. His main research interests are focused on the design and analysis of nonlinear oscillator based circuits, millimeter wave and terahertz antennas, circuits and systems, graphene based components, and textile integrated circuits and antennas. 


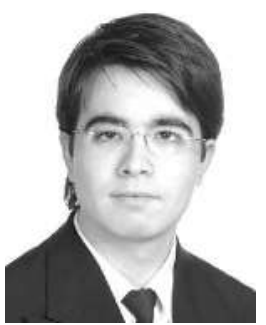

Carlos Vázquez-Antuña received the M.Sc. degree in telecommunication engineering, M.Sc. degree in information technology and mobile communications, and Ph.D. degree from the University of Oviedo, Gijón, Spain, in 2007, 2008, and 2013, respectively.

From 2007 to 2012, he was a Graduate Research Assistant, and since 2012, a Research Fellow with the Signal Theory and Communications Group, University of Oviedo. His research efforts mainly focus on nonlinear analysis and optimization techniques for the design of multifunctional oscillator-based circuits, active antennas and passive components, such as frequency multipliers and harmonic mixers, at microwave, millimeter/submillimeter-wave, and terahertz frequencies.

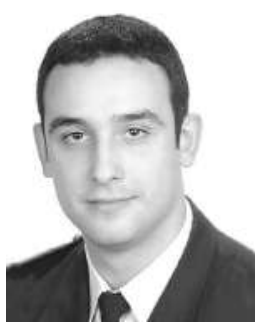

Miguel Fernández-García received the M.Sc. degree in telecommunication engineering, the M.Sc. degree in information technology and mobile communications and the Ph.D. degree from the University of Oviedo, Gijón, Spain, in 2006, 2010, and 2010, respectively.

From 2006 to 2008, he was a Research Fellow with the Signal Theory and Communications Group, University of Oviedo, where he has been an Associate Professor since 2008. His current research interests include nonlinear analysis and optimization techniques for the design of oscillator-based circuits, active antennas and frequency multipliers and mixers at the microwave, millimeter/submillimeterwave, and terahertz frequency bands.

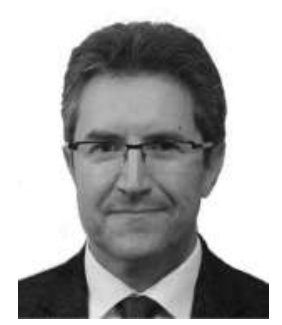

Fernando Las-Heras Andrés (M'86-SM'08) received the M.S. and Ph.D. degrees in telecommunication engineering from the Technical University of Madrid (UPM), Madrid, Spain, in 1987 and 1990, respectively.

He was a National Graduate Research Fellow from 1988 to 1990 and an Associate Professor from 1991 to 2000 with the Department of Signal, Systems and Radiocom, UPM. He was the Vice-Dean of Telecommunication Engineering with the Technical School of Engineering, Gijón, from 2004 to 2008. He was a Visiting Researcher with Syracuse University, New York, NY, USA and a Visiting Lecturer with the National University of Engineering, Lima, Peru, and the École Supérieure d'Ingénieurs en Génie Électrique, Rouen, France. He has been the Head with the Signal Theory and Communications Research Group, Department of Electrical Engineering, University of Oviedo, Gijón, Spain, since 2001, and has been a Full Professor since 2003. He has authored over 450 technical journal and conference papers in the areas of electromagnetic radiation, propagation and scattering theory and applications as well as inverse problems.

Dr. Las-Heras held the Telefónica Chair on "RF Technologies, ICTs Applied to Environment and Climate Change", and ICTs and Smartcities, from 2005 to 2015. He was a member of the Board of Directors of the IEEE Spain Section from 2012 to 2015, and a member of the Science, Technology and Innovation Council of Asturias, Spain, in 2010. He is a member of the IEEE Microwaves and Antennas Propagation Chapter (AP03/MTT17) Board from 2016 to 2018 